\title{
Effect of Static Magnetic Field on the Rate of Proliferation and Viability in HeLa Cancer Cells and Normal Fibroblasts
}

\section{ARTICLE INFO}

\section{Article Type}

Original Research

\section{Authors}

Shams E.* $B S C$

Javani Jouni F. ${ }^{1} P h D$,

Zafari J.2 $M S c$,

Monajemi R. ${ }^{3} P h D$

Abdolmaleki P. ${ }^{4} P h D$

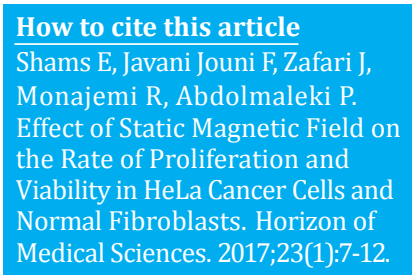

*Young Researchers and Elite Club, Felaverjan Branch, Islamic Azad University, Isfahan, Iran

${ }^{1}$ Microbiology Department, Tehran North Branch, Islamic Azad University, Tehran, Iran

2"Toxicology Research Center" and "Toxicology Department, Pharmacy School", Ahvaz Jundishapur University of Medical Sciences, Ahvaz, Iran

${ }^{3}$ Biology Department, Felaverjan Branch, Islamic Azad University, Isfahan, Iran

${ }^{4}$ Biophysics Department, of Biological Sciences Faculty, Tarbiat Modares University, Tehran, Iran

\section{Correspondence}

Address: Young Researchers and Elite Club, Islamic Azad University, Daneshgah Boulevard, Basij Boulevard, Falavarjan, Isfahan, Iran. Post Box: 155/84515 Phone: +98 (31) 37420134 Fax: +98 (31) 37432601 e.shams88@yahoo.com

\section{Article History}

Received: July 23, 2016

Accepted: September 7, 2016

ePublished: January 19, 2017

\section{A B S T R A C T}

Aims The increasing use of the electromagnetic devices in daily life leads to higher electromagnetic filed effects. The effects on the organic systems are contradictory and controversial. The aim of this study was to investigate the effects of different intensities and durations of the static magnetic fields on the living cells and their proliferation rate.

Materials \& Methods In the applied study, two HeLa cancer cell lines and human fibroblast natural cells were studied. At first, the cells were cultured on DMEN medium. Three magnetic intensities (7, 14, and 21T) and two durations (24 and 48h) were used, and the cells were treated by static magnetic field. The living cell percentage and cell proliferation rate were assessed by MTT method. Trypan blue was used in staining. And an optical microscope was used in enumeration. Data was analyzed by Graphpad Prism 5 using one-way ANOVA.

Findings The higher the static magnetic field and the more the duration were, the lesser the percentage of living cells and cell proliferation, showing a significant reduction in the HeLa cancer cells, while it was insignificant in the fibroblast natural cells. The highest reduction in the living cell percentage and cell proliferation rate was in 48-hour $21 \mathrm{~T}(\mathrm{p}<0.05)$.

Conclusion The static magnetic field affects the HeLa cancer cells more than the fibroblast cells. The higher the field intensity and the more the duration are, the lesser the alive cell percentage and cell proliferation rate.

Keywords Magnetic Field Therapy; HeLa Cells; Fibroblast

\section{CIT A T I O N L I N KS}

[1] Study of the influence of magnetic field on some biological characteristics of ... [2] Biological effects due to weak magnetic field on ... [3] Static and ELF magnetic fields induce tumor growth inhibition and ... [4] Static magnetic fields for the treatment ... [5] Effects of a static magnetic field on cell growth and gene expression in Escherichia ... [6] Teratogenic effects of static magnetic field on mouse ... [7] Rapporteur report: Cellular, animal and epidemiological studies of the effects of static magnetic fields relevant to human ... [8] Experimental evidence for $60 \mathrm{~Hz}$ magnetic fields operating through the signal transduction cascade: Effects on calcium influx and ... [9] Induction of stress proteins by electromagnetic fields in ... [10] Effects of magnetic field on the antioxidant enzyme activities of suspensioncultured tobacco ... [11] Cytotoxic effect of boswelliaserratahydroalcholic extract on human cervical carcinoma epithelial ... [12] In-vitro cytotoxicity activity of solanumnigrum extract against HeLa cell line and vero cell ... [13] A novel L1 retrotransposon marker for HeLa cell line ... [14] The Genomic and Transcriptomic Landscape of ... [15] Evaluation of anti-cancer effect of Peganumharmala L hydroalcholic extract on human cervical carcinoma epithelial ... [16] Cyclophosphamide-induced morphological changes in dental root development of ICR ... [17] Investigation of curcumin effects on liver tissue in adult male rats treated with ... [18] Studying the effect of static ... [19] The effect of low-frequency electromagnetic fields on some biological a ctivities of ... [20] Participation of target Fas protein in apoptosis pathway induced by CD4+ Th1 and CD8+ cytotoxic ... [21] Static magnetic field of 6 mT induces apoptosis and alters cell cycle in p53 mutant ... [22] Effect of $7 \mathrm{mT}$ static magnetic field and iron ions on rat lymphocytes: apoptosis, necrosis and ... [23] Investigation of the effects of static magnetic ... [24] Magnetic fields increase cell survival by ... [25] Effects of Electromagnetic Field on ... [26] Effects of electromagnetic field on ... [27] Investigation on the effect of static magneticfield up to ... [28] The effect of pulsed electromagnetic ... [29] Magnetic-field-induced DNA strand breaks in ... [30] Repetitive exposure to a 60-Hz ... 
ميدانهاى مغناطيسى خند دمهزار برابر ميدانى است كه انسان

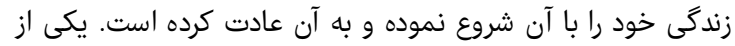

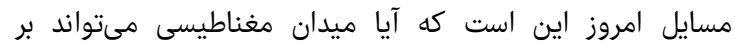

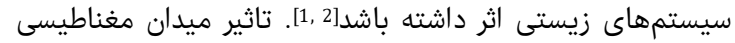

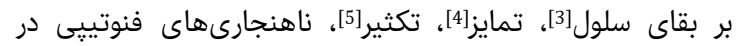

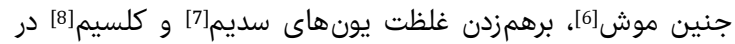

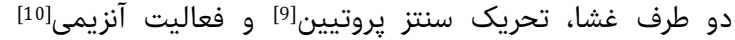
براسى شده است. سرطان در קند دها آنه اخير روند رو به افزايش داشته است. سرطان

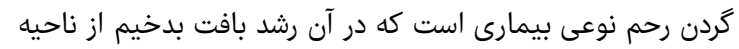

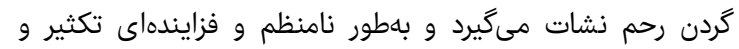

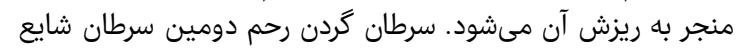

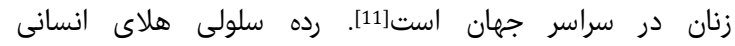

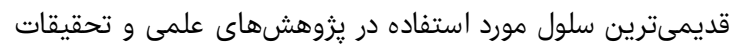

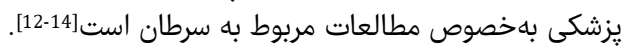

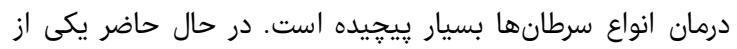

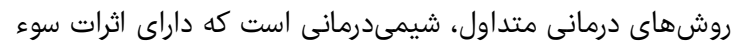

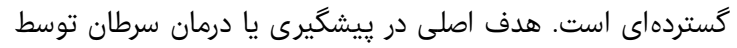

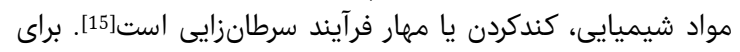

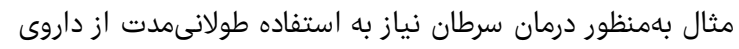

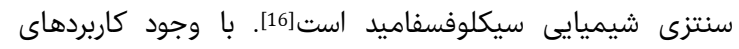

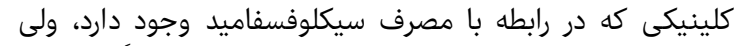

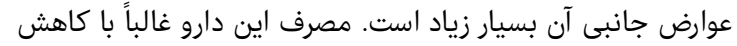

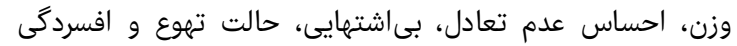

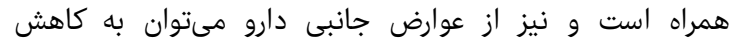

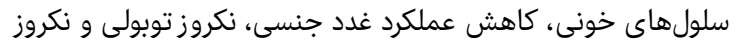
كَسترده ميوكارد اشاره كرد [17].].

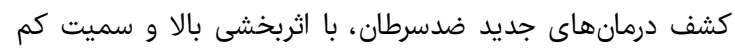

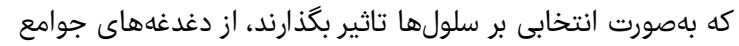

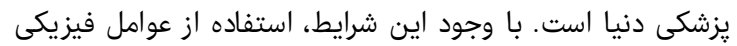

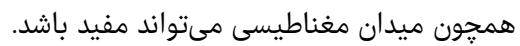

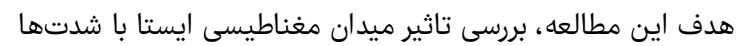

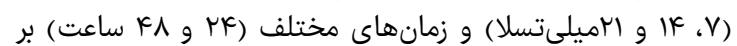
درصد سلولهاى زنده و نرخ تكثير آنها بود.

\section{مواد و روشها}

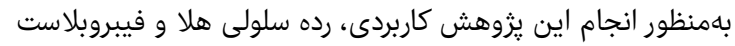

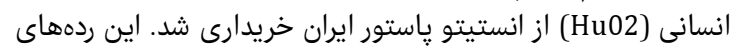

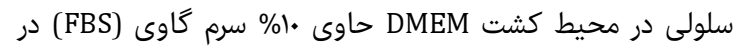

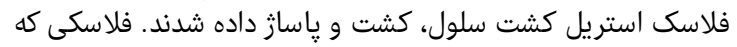

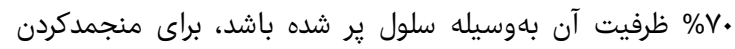

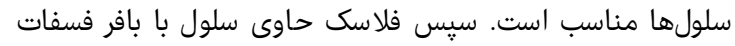

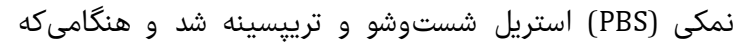

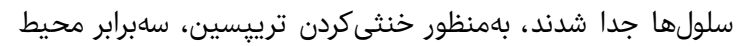

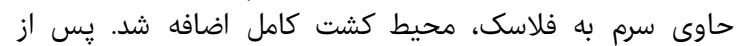

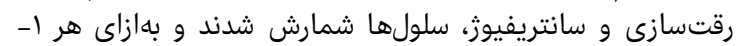

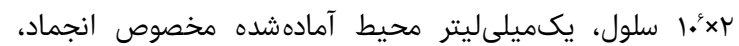

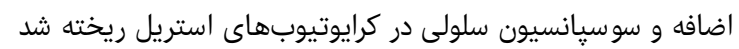

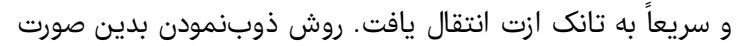

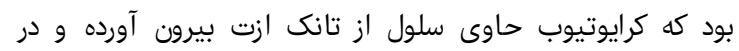

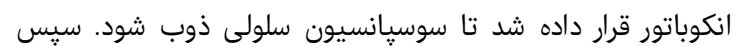

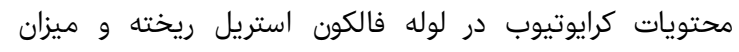

دوره سץ، شماره ا، زمستان موس|
اثر ميدان مغناطيسى ايستا بر نرخ تكثير و درصد

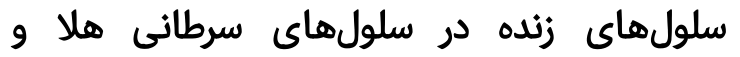
فيبروبلاستهاى طبيعى زونى درى سلون

الها شمس"

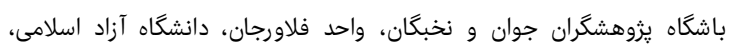
اصفهان، ايران

فاطمه جوانى جونى إنى كروه زيست شناسى سلولى و مولى مولكولى، واحد تهران شمال، دانشكاه آزاد اسلامى، تهران، ايران جابر ظفرى تهرى ايران "مركز تحقيقات سم شناسى" و و "كروه سمشناسى، دانشكده داروسازى"، دانشكاه علوم يزشكى جندى شايور اهواز، اهواز، ايران رامش منجمى PhD

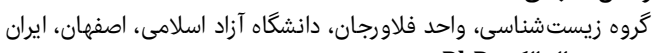

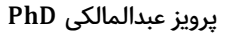
كَرِوه بيوفيزيك، دانشكده علوم زيستى، دانشكاه تربيت مدرس، تهران، ايران جكيده

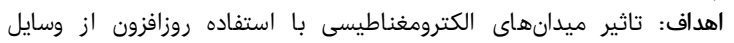

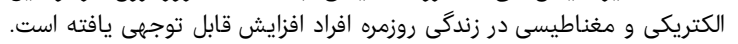

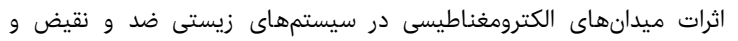

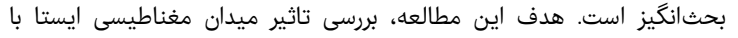

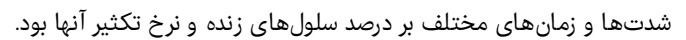

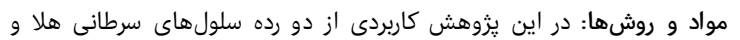

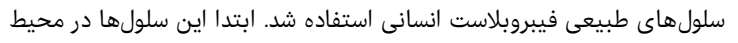

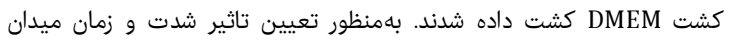

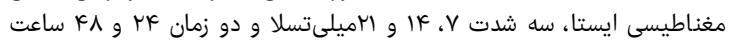

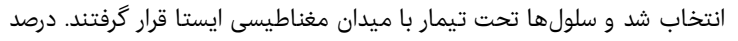

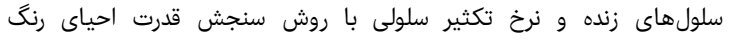

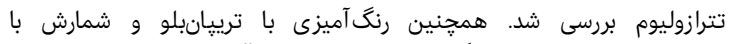

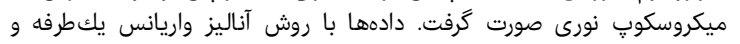

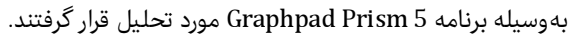

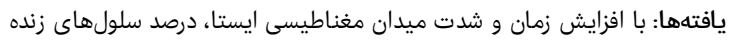

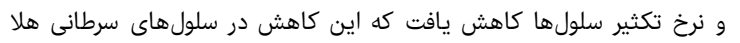

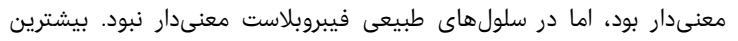

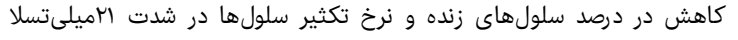

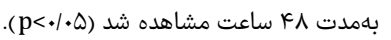

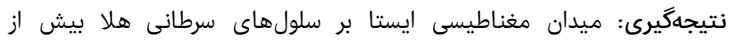

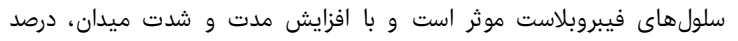

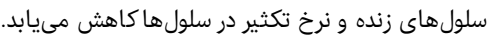

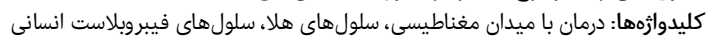

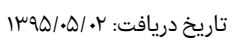

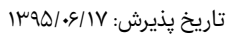
e.shams88@yahoo.com :نويسنده مسئور:

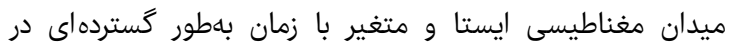

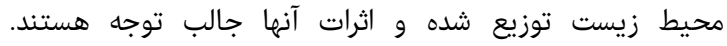

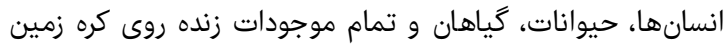

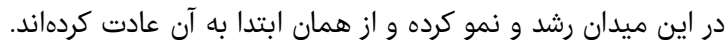

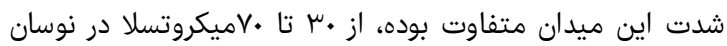

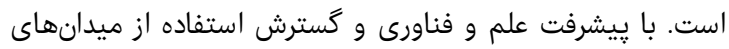

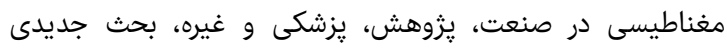

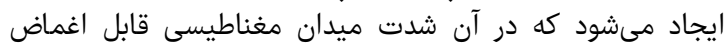

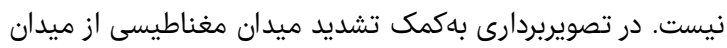

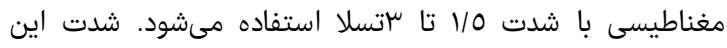




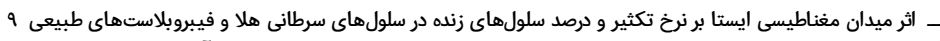

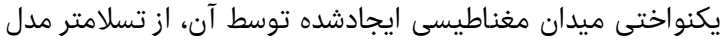

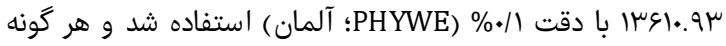

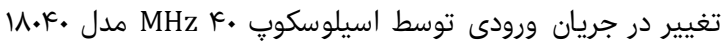
(Leader)

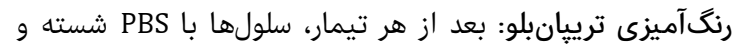

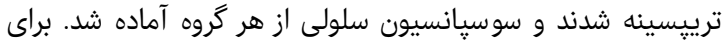

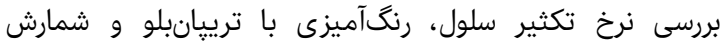

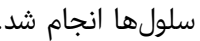

تحليل آمارى: دادهها با حداقل سه اردام تكرار مستقل بهصورت ميانكين

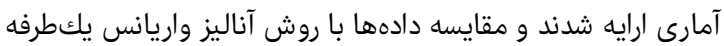

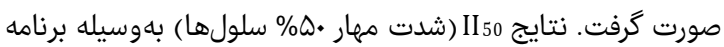
Graphpad Prism 5

يافتهها با افزايش زمان و شدت ميدان مغناطيسى ايستا، درصد سلولهاى كای

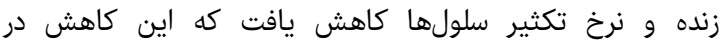

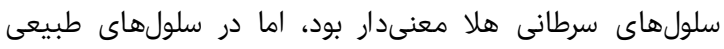

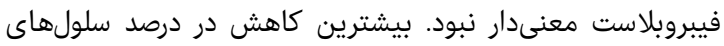

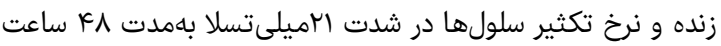

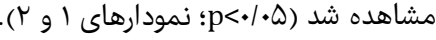

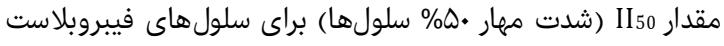

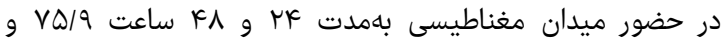

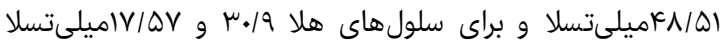

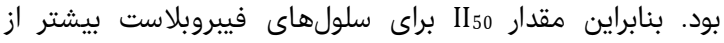

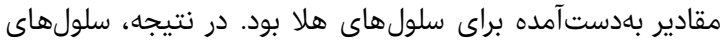

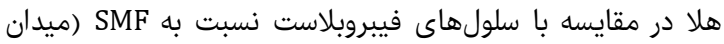
مغناطيسى ايستا) حساستر بودند.

بحث

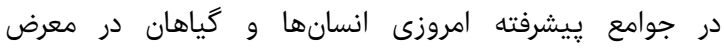

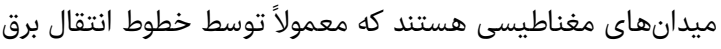

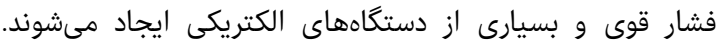

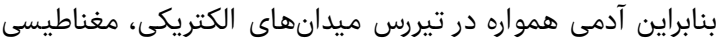

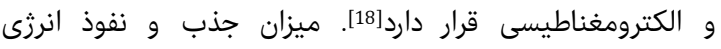

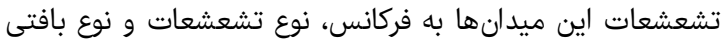

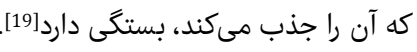

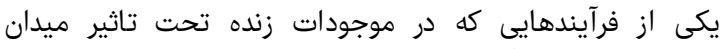

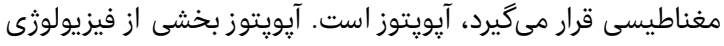

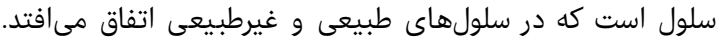

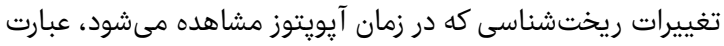

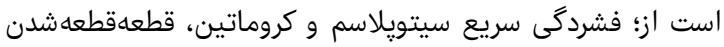
DNA

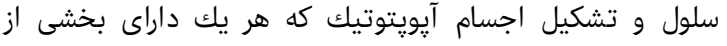

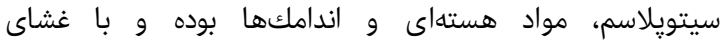

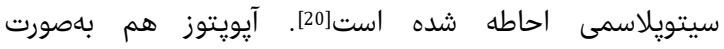

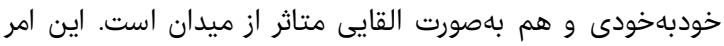

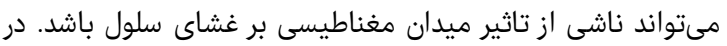

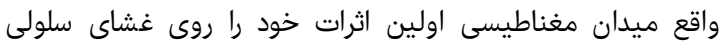

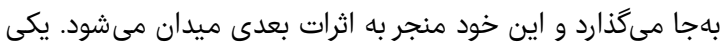

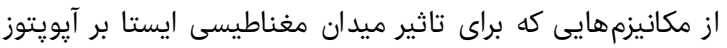

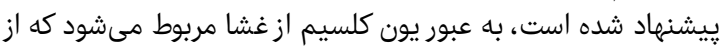

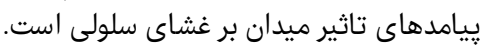

ساميلىليتر محيط DMEM فاقد بافر فسفات به آن اضافه و

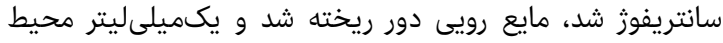

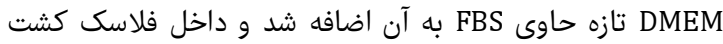

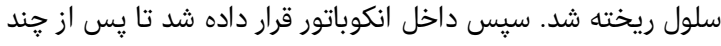

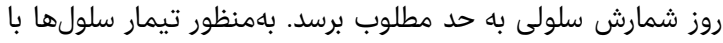

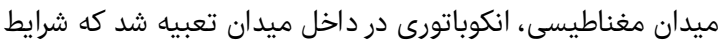

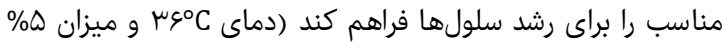
(CO2

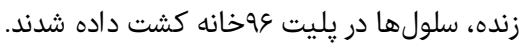

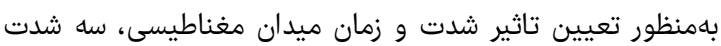

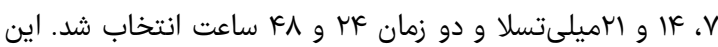

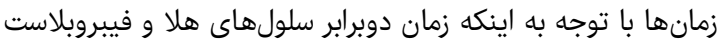

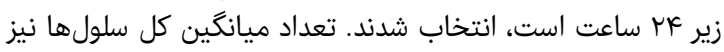

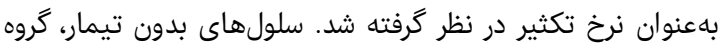

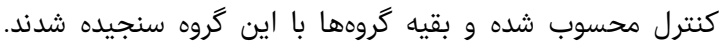

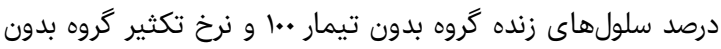

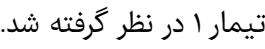

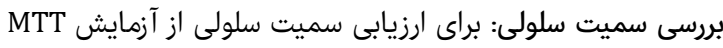

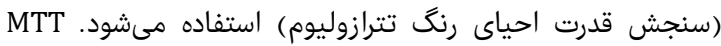

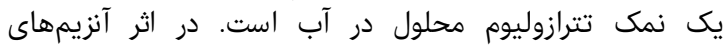

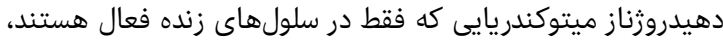

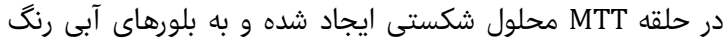

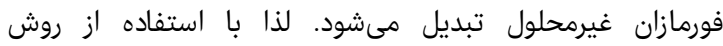
نورسنجى در طول موج • •

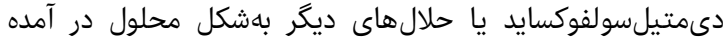

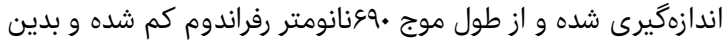

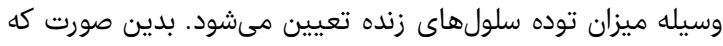

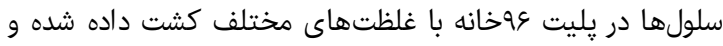

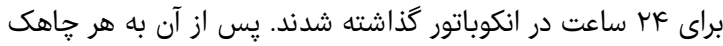

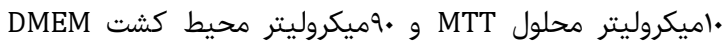

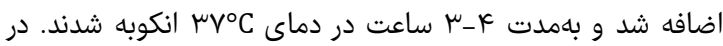

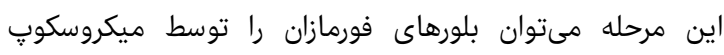

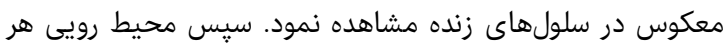

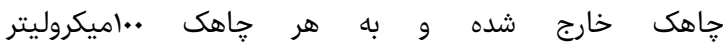

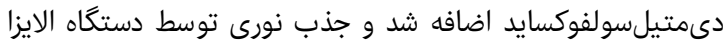

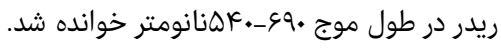
دستگاه مولد ميدان مغناطيسى ايستا: دستگًاه مولد مولد ميدان

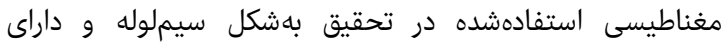

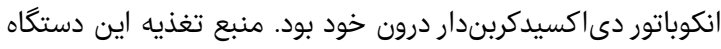

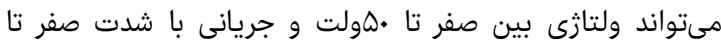

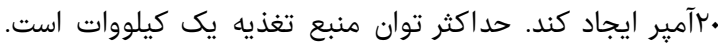

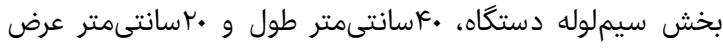

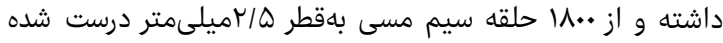

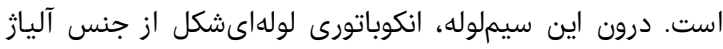

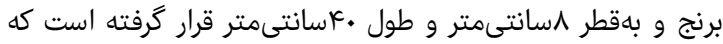

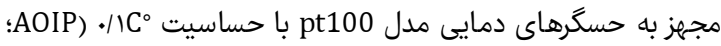

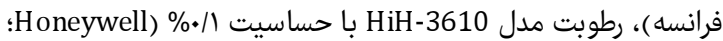

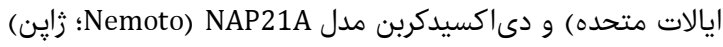

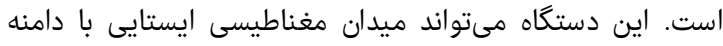

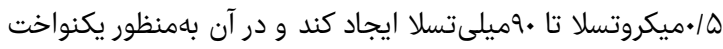

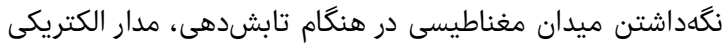

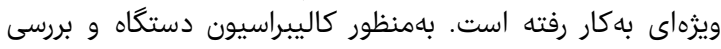




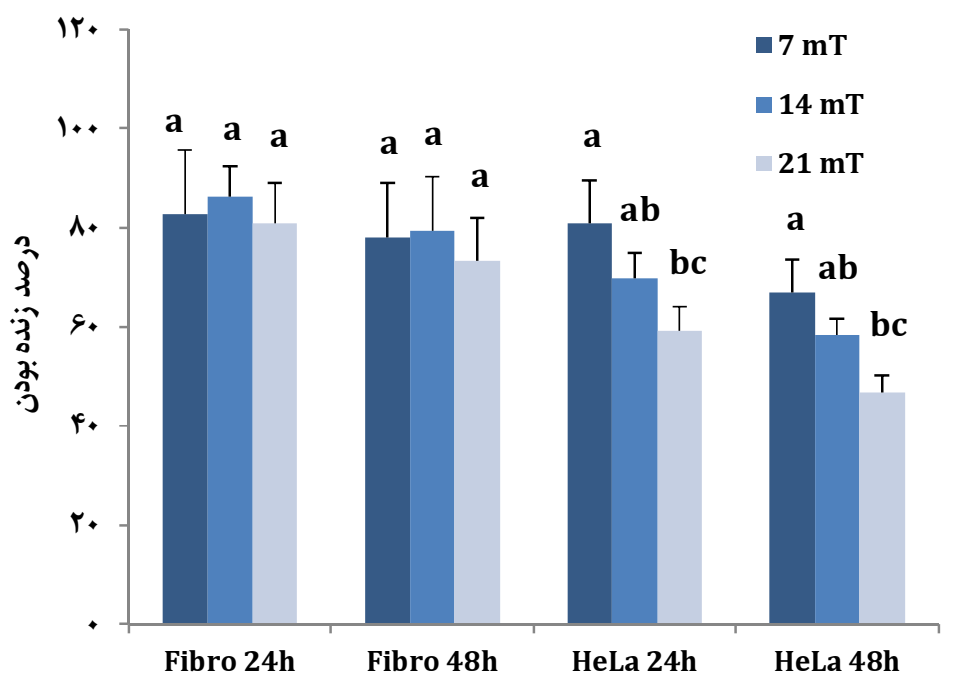

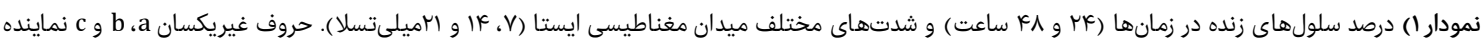

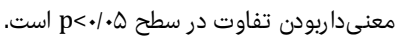

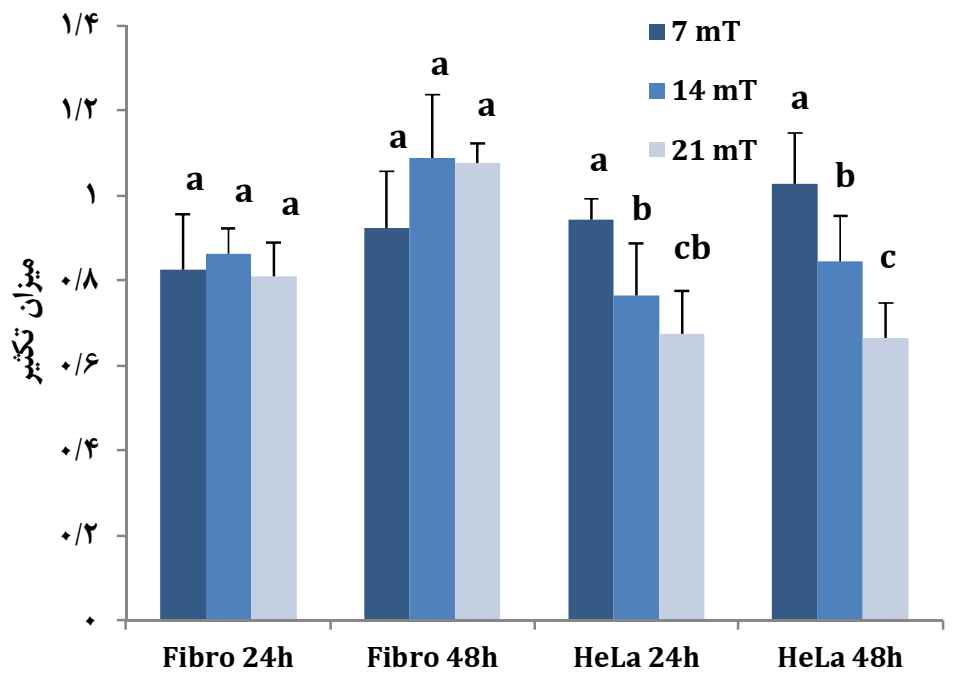

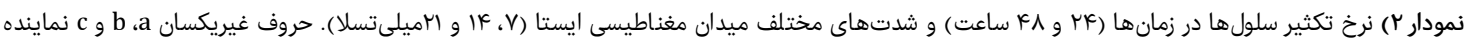

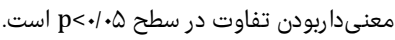

درون سلول، موجب آسيبرسانى به مولكولهاى DNA، لييبدها و

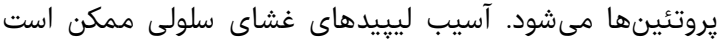

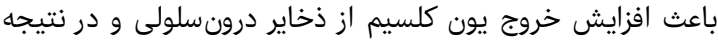
موجب كاهش يون كلسيم در سلولهایى ميدانديده شود [21].

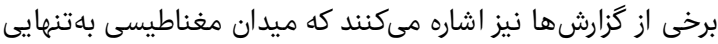

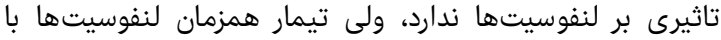

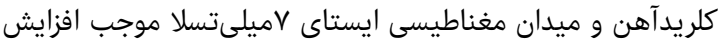

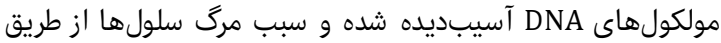

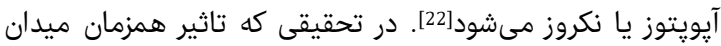

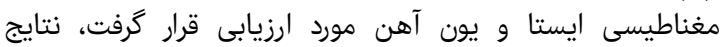

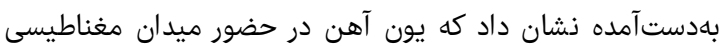

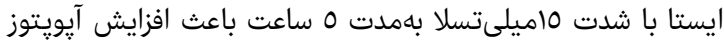

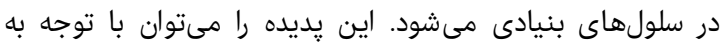

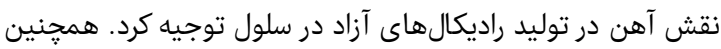

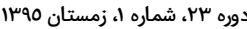

يزووهشهاى محققان يِيشين نشان مىدهد كه ميدان مغناطيسى

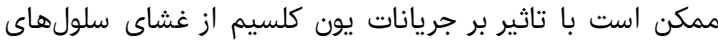

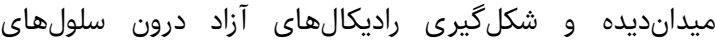
ميدانديده، روى درصد سلولهاي زنديده زيده، نرخ تكثير و آيويتوز سلول

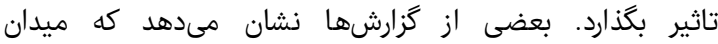

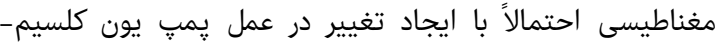
ATPase

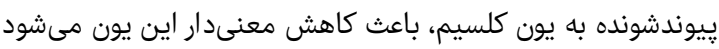

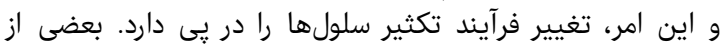

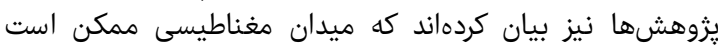

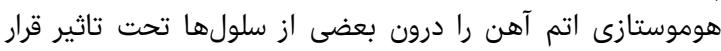

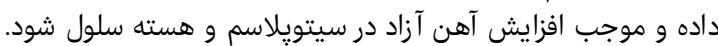

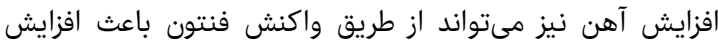

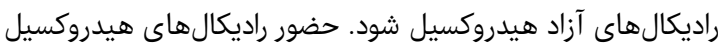




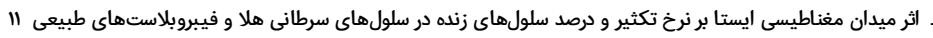

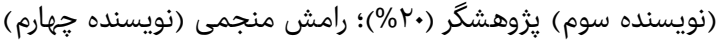

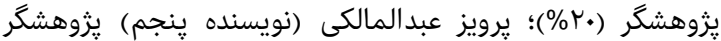

منابع مالى: اين تحقيق در قالب طرح يثزوهشى با شماره طرح

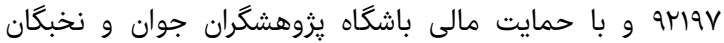

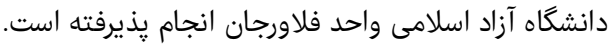

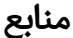

1- Aladjadjiyan A. Study of the influence of magnetic field on some biological characteristics of Zea mays. J Cent Eur Agric. 2002;3(2):89-94.

2- Belyavskaya NA. Biological effects due to weak magnetic field on plants. Adv Space Res. 2004;34(7):1566-74.

3- Tofani S, Barone D, Cintorino M, de Santi MM, Ferrara A, Orlassino R, et al. Static and ELF magnetic fields induce tumor growth inhibition and apoptosis. Bioelectromagnetics. 2001;22(6):419-28.

4- McLean M, Engström S, Holcomb R. Static magnetic fields for the treatment of pain. Epilepsy Behav. 2001;2(3):S74-S80.

5- Potenza L, Ubaldi L, De Sanctis R, De Bellis R, Cucchiarini L, Dachà $M$. Effects of a static magnetic field on cell growth and gene expression in Escherichia coli. Mutat Res. 2004;561(1-2):53-62.

6- Saito K, Suzuki H, Suzuki K. Teratogenic effects of static magnetic field on mouse fetuses. Reprod Toxicol. 2006;22(1):118-24.

7- Leszczynski D. Rapporteur report: Cellular, animal and epidemiological studies of the effects of static magnetic fields relevant to human health. Prog Biophys Mol Biol. 2005;87(2):247-53.

8- Liburdy RP, Callahan DE, Harland J, Dunham E, Sloma TR, Yaswen P. Experimental evidence for $60 \mathrm{~Hz}$ magnetic fields operating through the signal transduction cascade: Effects on calcium influx and c-MYC mRNA induction. FEBS Lett. 1993;334(3):301-8.

9- Pipkin JL, Hinson WG, Young JF, Rowland KL, Shaddock JG, Tolleson WH, et al. Induction of stress proteins by electromagnetic fields in cultured HL-60 cells. Bioelectromagnetics. 1999;20(6):347-57.

10- Sahebjamei H, Abdolmaleki P, Ghanati F. Effects of magnetic field on the antioxidant enzyme activities of suspension-cultured tobacco cells. Bioelectromagnetics. 2007;28(1):42-7.

11- Forouzandeh S, Naghsh N, Salimi S, Jahantigh D. Cytotoxic effect of boswellia serrata hydroalcholic extract on human cervical carcinoma epithelial cell line. Med Lab J. 2014;8(1):7-13. [Persian]

12- Patel S, Gheewala N, Suthar A, Shah A. In-vitro cytotoxicity activity of solanum nigrum extract against HeLa cell line and vero cell line. Int J Pharm Pharm Sci. 2009;1(Suppl 1):38-46.

13- Rahbari R, Sheahan T, Modes V, Collier P, Macfarlane C, Badge RM. A novel L1 retrotransposon marker for HeLa cell line identification. Biotechniques. 2009;46(4):277-84.

14- Landry JJ, Pyl PT, Rausch T, Zichner T, Tekkedil MM, Stütz AM, et al. The Genomic and Transcriptomic Landscape of a HeLa Cell Line. G3 (Bethesda). 2013;3(8):1213-24.

15- Forouzandeh F, Salimi S, Naghsh N, Zamani N, Jahani S. Evaluation of anti-cancer effect of Peganum harmala L
در اين بررسى مشخص شد كه يون آهن بهتنهايى و بدون ميدان

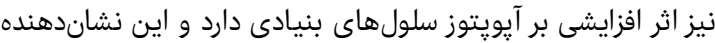

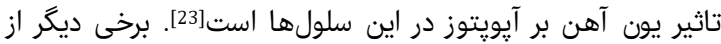

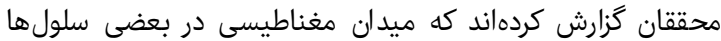

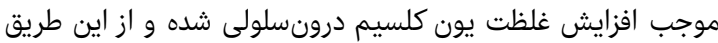

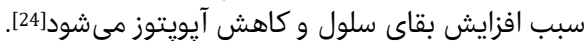

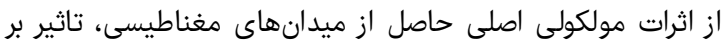

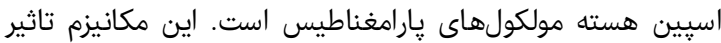

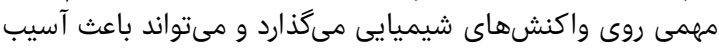

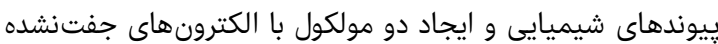

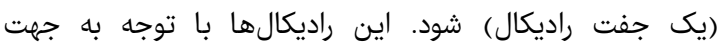

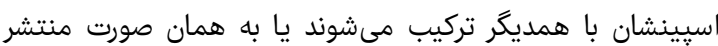

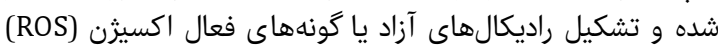

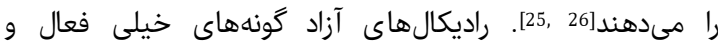

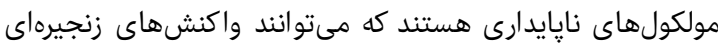

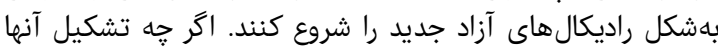

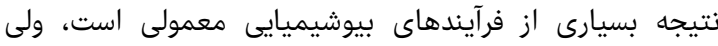
عملكرد آنها بسيار زيان آور است.

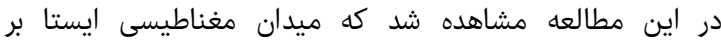

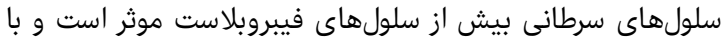

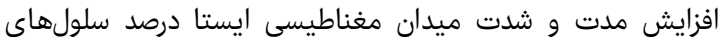

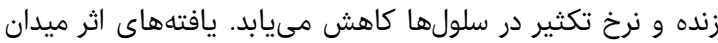

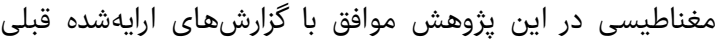

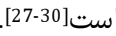

از محدوديتهاى اين مطالعه مىتوان به دستگًاه مولد ميدان

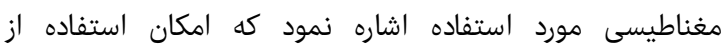

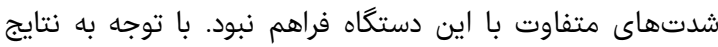

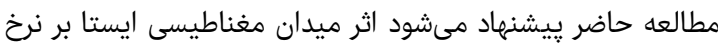

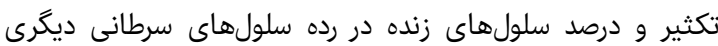

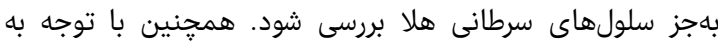

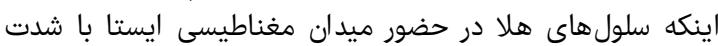

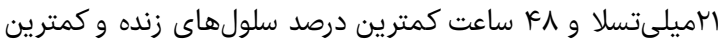

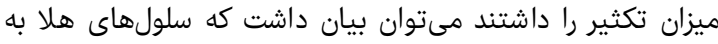

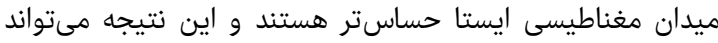

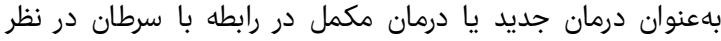

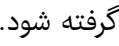

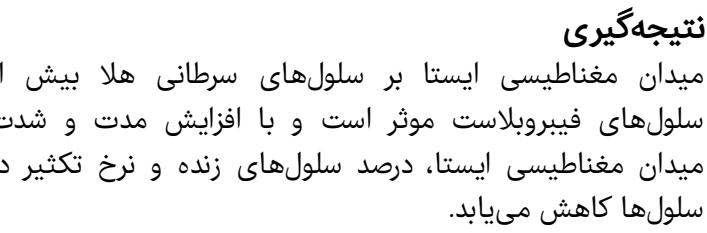

تشكر و قدردانى: نويسندكان اين مقاله بر خود لازم مىدانند از

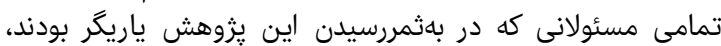

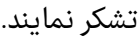
تاييديه اخلاقى: هر گُونه مورد اخلاقى است.

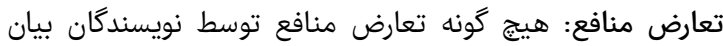

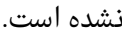

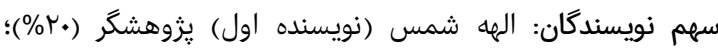

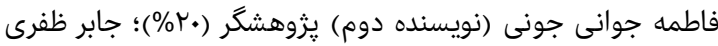


magnetic field on apoptosis in bone marrow stem cells of rat. Environ. 2009;29(2):220-4.

24- Fanelli C, Coppola S, Barone R, Colussi C, Gualandi G, Volpe $P$, et al. Magnetic fields increase cell survival by inhibiting apoptosis via modulation of $\mathrm{Ca}^{2+}$ influx. FASEB J. 1999;13(1): 95-102.

25- Kula B, Sobczak A, Kuska R. Effects of Electromagnetic Field on Free-Radical Processes in Steelworkers. Part I: Magnetic Field Influence on the Antioxidant Activity in Red Blood Cells and Plasma. J Occup Health. 2002;44(4):226-9

26- Sobczak A, Kula B, Danch A. Effects of electromagnetic field on free-radical processes in steelworkers. Part II: Magnetic field influence on vitamin $\mathrm{A}, \mathrm{E}$ and selenium concentrations in Plasma. J Occup Health. 2002;44(4):230-3.

27- Javani Jouni F, Abdolmaleki P, Movahedin M. Investigation on the effect of static magnetic field up to $15 \mathrm{mT}$ on the viability and proliferation rate of rat bone marrow stem cells. In Vitro Cell Dev Biol Anim. 2013;49(3):212-9.

28- Aldinucci C, Palmi M, Sgaragli G, Benocci A, Meini A, Pessina $F$, et al. The effect of pulsed electromagnetic fields on the physiologic behaviour of a human astrocytoma cell line. Biochim Biophys Acta. 2000;1499(1-2):101-8.

29- Lai H, Singh NP. Magnetic-field-induced DNA strand breaks in brain cells of the rat. Environ Health Perspect. 2004;112(6):687-94.

30- Kim J, Ha CS, Lee HJ, Song K. Repetitive exposure to a 60-Hz time-varying magnetic field induces DNA doublestrand breaks and apoptosis in human cells. Biochem Biophys Res Commun. 2010;400(4):739-44. hydroalcholic extract on human cervical carcinoma epithelial cell line. J Shahrekord Univ Med Sci. 2014;16(4):1-8. [Persian]

16- Kawakami T, Nakamura Y, Karibe $H$. Cyclophosphamide-induced morphological changes in dental root development of ICR mice. PLoS One. 2015;10(7):e0133256.

17- Khodaparast Z, Yousofi AR, Khoshvagti A. Investigation of curcumin effects on liver tissue in adult male rats treated with cyclophosphamide. J Fasa Univ Med Sci. 2014;4(3):344-52. [Persian]

18- Sabet A, Abdolmaleki P, Mowla SJ, Ghanati F. Studying the effect of static magnetic field on induced apoptosis in mesenchymal bone marrow stem cells of rat. Exp Animal Biol. 2013;1(2):17-23. [Persian]

19- Baharara J, Zahedifar Z. The effect of low-frequency electromagnetic fields on some biological a ctivities of animals. Arak Med Univ J. 2012;15(7):80-93. [Persian] 20- Ju S-T, Cui H, Panka DJ, Ettinger R, MarshakRothstein A. Participation of target Fas protein in apoptosis pathway induced by CD4+ Th1 and CD8+ cytotoxic $\mathrm{T}$ cells. Proc Natl Acad Sci U S A. 1994;91(10):4185-9.

21- Ahmadianpour MR, Abdolmaleki P, Mowla SJ, Hosseinkhani S. Static magnetic field of $6 \mathrm{mT}$ induces apoptosis and alters cell cycle in p53 mutant Jurkat cells. Electromagn Biol Med. 2013;32(1):9-19.

22- Jajte J, Grzegorczyk J, Zmyślony M, Rajkowska E. Effect of $7 \mathrm{mT}$ static magnetic field and iron ions on rat lymphocytes: apoptosis, necrosis and free radical processes. Bioelectrochemistry. 2002;57(2):107-11.

23- Tavasoli Z, Abdolmaleki P, Mowla SJ, Ghanati F, Sarvestani AS. Investigation of the effects of static 